\title{
Perinatal Risk Factors and Early Onset of Neonatal Sepsis
}

\author{
Fedaa Noah Noah, MD, Leen Jamel Doya, MD* (D) and Oday Jouni \\ Department of Pediatrics, Faculty of Medicine, Tishreen University Hospital, Latakia, Syria
}

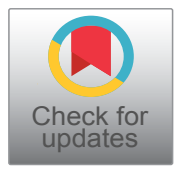

*Corresponding author: Leen Jamel Doya, MD, Department of Pediatrics, Faculty of Medicine, Tishreen University Hospital, Latakia, Syria, Tel: 963992856983

\begin{abstract}
Background: Neonatal sepsis contributes significantly to neonatal morbidity and mortality and is an ongoing major global public health challenge particularly in developing countries.

Objective: The study aims to determine the prevalence of early neonatal infections and risk factors associated with neonatal intensive care.

Methods: In a cross-sectional study that included all newborns admitted to the neonatal intensive care of Tishreen University Hospital from October 2019 for one year and who fulfilled clinical and laboratory criteria for early neonatal sepsis, blood samples were drawn for laboratory analysis (CBC, CRP) with a blood culture.

Results: 197 neonates (28.14\%) had early neonatal sepsis. The majority of patients $(80.71 \%)$ had negative blood cultures. The most common pathogen of the early neonatal infection was Staphylococcus albicans, Streptococci, and E. coli. The current study found that the most prevalent risk factor for early neonatal infection was a cesarean section, followed by maternal infections, male newborn sex, low birth weight, prematurity, maternal age greater than 30 years and less than 20 years, early rupture of membranes, need for resuscitation, and Meconium amino fluid.

Conclusion: The current study confirmed an important prevalence of early neonatal sepsis in Tishreen University Hospital with its association with many risk factors; the most prevalent factors were cesarean section, the gender of the male newborn, low birth weight, and prematurity.
\end{abstract}

\section{Keywords}

Early neonatal sepsis, Risk factors, Cesarean delivery, Maternal sepsis

\section{Background}

Early neonatal septicemia is a clinical laboratory syndrome caused by the passage of pathogens, their toxins, or their antigens into the blood circulation during the first 72 hours of life [1]. The diagnosis is made clinically by non-specific manifestations (low or high temperature of the newborn, lethargy, irritability, tachypnea or episodes of apnea, rapid or weak pulse, hypotension, low or high blood sugar, metabolic acidosis) [2].

In developing countries, the incidence of neonatal septicemia is $1.6 \%$ of all live births [3]. Early and late neonatal septicemia is the most common problem in the newborn stage that caused high morbidity and mortality rate. It is responsible for (30-50)\% of neonatal deaths in developing countries, according to WHO estimates [4].

The pathogens that cause early neonatal sepsis are transmitted through the placenta, during childbirth, or after childbirth [5]. It may be bacterial, viral, or fungal. Streptococcus (group B) is the most common pathogen that causes early neonatal sepsis (50\%), followed by E. coli (30\%), then Listeria, Haemophilus influenza, Enterococci, Staphylococci, and Pneumococci [6].

There are many risk factors for early neonatal sepsis as maternal factors (urinary and genital infections, prenatal fever, prenatal laboratory septicemia, multiple pregnancies, frequent vaginal examinations, premature rupture of membranes more than 18 hours), neonatal factors (prematurity, low birth weight, perinatal asphyxia, low Apgar scores, intrauterine infection), environmental factors (use of resuscitation tools, medical and nursing staff) [7]. White blood cells (WBC), neutrophil count, C-reactive protein (CRP), and platelet count (PLT) are the laboratory criteria for early neonatal sepsis. Blood culture is the gold standard in diagnosis [8]. 
Despite the great development and progress in neonatal treatment, high mortality rates (30-50\%) are still a concern in developing countries compared to developed countries.

No local study has determined the prevalence of risk factors for early neonatal sepsis in Syria, so we conducted this research to find the most common risk factors for sepsis.

\section{Material and Methods}

The current study was reviewed and approved by the ethical committee of Tishreen University Hospital. Informed consent was obtained from the patient's parents.

An observational descriptive study included 197 neonates who were referred toneonatal intensive care at Tishreen University Hospital in Lattakia over 1 year between October 2019 to November 2020.

On admission, detailed information was recorded on the research form (age, sex, gestational age, birth weight, age at admission, type of delivery, type of pregnancy, use of assistive devices, premature rupture of membranes, maternal age, urinary and genital infections in the mother, laboratory evaluation of the mother, amniotic fluid, obstetric Apgar, need for postpartum resuscitation, presence of congenital anomalies) with a full clinical examination of the patients included in the study. WBC, CRP, PLT with a blood culture procedurewere performed in the laboratory of Tishreen University Hospital.

\section{Statistical methods}

All data were analyzed using Statistical Package for social sciences (SPSS version 20). Descriptive statistical parameters (mean and standard deviation, frequencies, and percentage) were calculated for each quantitative variable. The prevalence rate was calculated.

\section{Results}

The current study included 197 (28.14\%) of the neonates of 700 neonates who were referred to the neonatal intensive care at Tishreen University Hospital in Lattakia.

The blood culture was negative $(80.71 \%)$ in the majority of patients; staphylococcal infection (34.21\%), followed by streptococci (31.58\%), E. coli $(18.42 \%)$, and staphylococcus aureus (15.79\%). The majority of patients in the current study $(80.20 \%)$ were diagnosed with sepsis at an age less than 24 hours as shown in Table 1.

Most of the patients were males (65.5\%) with a statistically significant difference between the studied groups according to the age ( $p$-value $=0.004$ ) with Sex Ratio $(F: M)=1.9: 1$. Prematurity and low birth weight were $50.76 \%, 55.84 \%$ respectively in the early neonatal sepsis (Table 1).

24 patients (12.18\%) had congenital malformation; gastrointestinal malformations were the majority (58.33\%) (diaphragmatic atresia 1 patient, esophageal atresia 3 patients, intestinal atresia 1 patient, anal atresia 8 patients, Hirschsprung 1 patient), cardiac malformations were (20.83\%) (patent ductus arteriosus 1 patient, multiple cardiac malformation1 patient, ventricular aplasia 1 patient, foramen ovale 1 patient, common stump arterial 1 patient), neurologic malformations $12.44 \%$ (Hydrocephalus 2 patient, meningocele1 patient), urological malformation $4.2 \%$ (Bladder inversion 1 patient), extra fingers 1 patient.

The majority of the age of the mothers of the current study was more than 30-years-old (49.24)\%. Most of the patients' mothers $68.02 \%$ suffered from prenatal infections (respiratory $30.46 \%$, urinary $35.53 \%$, gastrointestinal 2.03\%). Most of the patients had sterilized amniotic fluid $86.29 \%$ (Table 2).

Table 1: The neonatal risk factors of the early onset of neonatal sepsis.

\begin{tabular}{|c|c|c|c|}
\hline \multicolumn{2}{|c|}{ The risk factor } & \multirow{2}{*}{$\begin{array}{l}\text { The number } \\
158\end{array}$} & \multirow{2}{*}{\begin{tabular}{|l} 
Percentage \\
$80.20 \%$ \\
\end{tabular}} \\
\hline \multirow{3}{*}{ Age } & Less than 24 hour & & \\
\hline & [24-48] hour & 15 & $7.6 \%$ \\
\hline & [48-72] hour & 24 & $12.2 \%$ \\
\hline \multirow[t]{2}{*}{ Sex } & male & 129 & $65.5 \%$ \\
\hline & female & 68 & $34.5 \%$ \\
\hline \multirow{4}{*}{ Gestational Age } & More than $37 \mathrm{GA}$ & 97 & $49.24 \%$ \\
\hline & [34-37] GA & 57 & $28.93 \%$ \\
\hline & [28-34[ GA & 39 & $19.8 \%$ \\
\hline & Less than $28 \mathrm{GA}$ & 4 & $2.03 \%$ \\
\hline \multirow{3}{*}{ Birth weight } & {$[1500-2500] \mathrm{g}$} & 66 & $33.5 \%$ \\
\hline & Less than $1500 \mathrm{~g}$ & 44 & $22.34 \%$ \\
\hline & More than $2500 \mathrm{~g}$ & 87 & $44.16 \%$ \\
\hline \multirow[b]{2}{*}{ Malformations } & Yes & 24 & $12.18 \%$ \\
\hline & No & 173 & $87.82 \%$ \\
\hline
\end{tabular}


Table 2: The mother risk factors of the early onset of neonatal sepsis.

\begin{tabular}{|c|c|c|c|c|}
\hline \multicolumn{3}{|c|}{ The risk factor } & The number & Percentage \\
\hline \multirow{3}{*}{ Mother age } & \multicolumn{2}{|c|}{ Less than $20 \mathrm{yr}$} & 20 & $10.15 \%$ \\
\hline & \multicolumn{2}{|c|}{ [24-30] yr } & 80 & $40.61 \%$ \\
\hline & \multicolumn{2}{|c|}{ More than $30 \mathrm{yr}$} & 97 & $49.24 \%$ \\
\hline \multirow[t]{2}{*}{ Mother infections } & \multicolumn{2}{|l|}{ Yes } & 134 & $68.02 \%$ \\
\hline & \multicolumn{2}{|l|}{ No } & 63 & $31.98 \%$ \\
\hline \multirow[t]{2}{*}{ Meconium-stained amniotic fluid } & \multicolumn{2}{|l|}{ No } & 170 & $86.29 \%$ \\
\hline & \multicolumn{2}{|l|}{ Yes } & 27 & $13.71 \%$ \\
\hline \multirow{3}{*}{$\begin{array}{l}\text { Premature rupture of } \\
\text { membranes }\end{array}$} & \multirow[t]{2}{*}{ Yes } & Less than $18 \mathrm{~h}$ & 25 & $12.69 \%$ \\
\hline & & More than $18 \mathrm{~h}$ & 61 & $30.96 \%$ \\
\hline & \multicolumn{2}{|l|}{ No } & 111 & $56.35 \%$ \\
\hline \multirow[t]{2}{*}{ Delivery mode } & \multicolumn{2}{|c|}{ Caesarean } & 168 & $85.28 \%$ \\
\hline & \multicolumn{2}{|c|}{ Vaginal } & 29 & $14.72 \%$ \\
\hline
\end{tabular}

The majority of patients $(56.35 \%)$ of the mothers did not have premature rupture of membranes, while $30.96 \%$ of the mothers had premature rupture of membranes more than 18 hours. Cesarean section was the most common type of delivery (85.28\%) (Table 2).

135 neonates $(68.53 \%)$ from the study sample did not require any resuscitation procedure, while 62 patients required resuscitation (31.47\%) [49 patients received Ambuventilation only (24.87\%), 13 patients were intubated with Ambuventilation (6.60\%)].

\section{Discussion}

The current study included 197 neonates (28.41\%) who were referred to neonatal intensive care at Tishreen University Hospital in Lattakia.

In the current study, the majority of blood cultures were negative $(80 \%)$, this may be that mothers in the majority of the sample had received prenatal preventive antibiotics in addition to the randomized use of antibiotics in our society. This is consistent with the results of many international studies in which positive blood cultures were recorded in only $30 \%$ of newborns with early septicemia [9].

In 2019, Abdul Rahman's study in Saudi Arabia on 245 newborns conducted that the largest cause of early newborn sepsis was Streptococcus (33.31\%), followed by E. coli (27.31\%). This is consistent with the Giannoni study $[10,11]$. In contrast to our study, which found the majority of blood cultures were $S$. albicans infection (34.21\%) followed by Streptococcus (31.59\%), and E. coli $(18.42 \%)$ this may be that the majority of patients were premature infants with the presence of intravenous catheters or it may be contamination when the sample is taken for culture or during blood culture.

The Caesarean section was (85.28\%) in the current study. This percentage is very high compared with zelalem study in Ethiopia in 2019, and the Asia Jabiri study in Dar AL Salaam Tanzaniain 2015. This may be attributed to a large number of cesarean sections without a real indication, due to the mothers' desire for rapid delivery, in addition to the lack of awareness of the risks related to this procedure $[12,13]$.

Maternal infection was (68.02\%) which is much higher than Giannoni's study in Switzerland $(8 \%)$ and Zelalem study in Ethiopia in 2019 (16.7\%) [10,13]. This discrepancy may be due to the different maternal health care systems applied in each country, as well as the economic, social and cultural factors in each country.

The percentage of the male sex as a risk factor for early neonatal sepsis was $(65.5 \%)$, while in both the Gianoni and Getabelew studies in Ethiopia it was $52 \%$ and $58.1 \%$, respectively, where the male sex is associated with 3.7 times higher risk of early neonatal sepsis than the feminine sex, the mechanism is not fully defined and is not clear. It is likely to be multifactorial with genetic, immunological, and hormonal influences. There are related factors such as genes linked to the $X$ chromosome in the female immune system $[10,13,14]$.

Low birth weight and preterm birth accounted for $55.84 \%$ and $50.76 \%$, respectively, of the study sample, which is similar to the Mate Siakwa studies in Ghana and Giannini in Switzerland. Birth weights are most likely to receive intravenous fluids and medications, as well as a lack of IgG immunoglobulins in premature infants, are transmitted to the fetus from the mother through the placenta primarily during the third trimester of pregnancy $[10,15,16]$.

Maternal age (above 30 years) and (less than 20 years) is considered a risk factor for early neonatal sepsis. The percentage of mothers over 30 years of age was the majority of patients with early neonatal sepsis $(49.50 \%)$ and this is close to most international studies such as the Ethiopian study in 2019 and Dar's study Salam, who confirmed that newborns of mothers over 30 years of age are more prone to early neonatal sepsis $[10,12]$. 
Hassan's study in Bangladesh showed that the incidence of early neonatal sepsis increases in the newborns of mothers less than 20 years of age (67\%) compared to our study, where it found that the rate is $10.15 \%$. This could be due to the difference in the sample size and the social situation in Bangladesh, which dictates that females marry at an early age $[8,11]$.

Maternal age less than 20 years is associated with a higher colonization rate of the vaginal wall with GBS(Group B Streptococcus), which explains the higher possibility of early neonatal sepsis, while age greater than 35 years is associated with the occurrence of serious medical complications associated with pregnancy such as gestational hypertension, diabetes, cardiovascular disease, congenital malformations and chromosomal problems, multiple pregnancies, prematurity, low birth weight and the need for cesarean section $[3,11]$.

The number of newborns diagnosed with early sepsis with premature rupture of membranes was about $43.65 \%$, which is close to what was found in the study of Asia Jabiri in Tanzania (49.5\%), whereby premature rupture of membranes may increase the risk of fetal exposure to pathogens in an ascending way [12].

The percentage of neonates who required resuscitation with early neonatal sepsis was (31.47\%), which is close to the percentage found by the Asia Jabiri study in Tanzania (37.7\%) [12].

Resuscitation at birth was also associated with the development of early sepsis in both the Mate Siakwa studies in Ghana and Getabelw in Ethiopia, as resuscitation using non-sterile equipment increases the likelihood of introducing pathogens into the lungs of a fetus whose immune system is not yet well developed $[14,15]$.

Both the Getabelw studies in Ethiopia and Saleh in Lattakia confirmed that meconium-stained amniotic fluid is a risk factor for early neonatal sepsis, $7 \%$, and $10 \%$, respectively. The same result was found in our study where the percentage reached $(13.71 \%)$ as sepsis is considered among the factors of fetal distress that often lead to a condition of meconium-stained amniotic fluid [11,14-17].

\section{Conclusion}

The current study showed an important prevalence of early neonatal sepsis in newborns admitted to neonatal intensive care at Tishreen University Hospital. It is associated with many risk factors such as cesarean section, prematurity, low birth weight in addition to the male gender.

\section{Data Availability}

We can't share patient data due to our hospital's privacy policy, which is concerned with maintaining patient confidentiality and refuses to publish or share data. Also, the informed consent signed by the parents to participate in the study prevents the sharing of information with the non-Study researchers.

\section{Acknowledgments}

We wish to thank the medical officer, doctors in the pediatric department and laboratory assistants in Tishreen University Hospital laboratory who worked with us in reviewing the patients and doing the laboratory analysis.

\section{Statement of Ethics}

All parents whose children were studied gave informed consent for sharing of this research. Ethical clearance for this study was obtained from the Ethical Committee of the University of Tishreen Hospital.

\section{Funding Sources}

None.

\section{Conflict of Interest Statement}

None declared.

\section{Author Contributions}

Both authors developed and carried out sample collection. Literature review, the data analysis and read through the final data were done by both authors.

\section{References}

1. Vergnano S, Menson E, Kennea N, Embleton N, Russel AB, et al. (2011) Neonatal infections in England: The NeonIN surveillance network. Arch Dis Child Fetal Neonatal Ed 96: F9-F14.

2. Kucova P, Kantor L, Fiserova K, Lasak J, Röderova M, et al. (2021) Bacterial pathogens and evaluation of a cut-off for defining early and late neonatal infection. Antibiotics 10 : 278 .

3. Lehtonen L, Gimeno A, Parra-Llorca A, Vento M (2017) Early neonatal death: A challenge worldwide. Semin Fetal Neonatal Med 22: 153-160.

4. Kim F, Polin RA, Hooven TA (2020) Neonatal sepsis. BMJ 1: 371-380.

5. Melvan JN, Bagby GJ, Welsh DA, Nelson S, Zhang P (2010) Neonatal sepsis and neutrophil insufficiencies. Int Rev Immunol 29: 315-348.

6. Shane AL, Sánchez PJ, Stoll BJ (2017) Neonatal sepsis. The lancet 390: 1770-1780.

7. Wynn JL (2016) Defining neonatal sepsis. Curr Opin Pediatr 28: $135-140$.

8. Wu JH, Chen CY, Tsao PN, Hsieh WS, Chou HC (2009) Neonatal sepsis: A 6-year analysis in a neonatal care unit in Taiwan. Pediatr Neonatol 50: 88-95.

9. Mersha A, Worku T, Shibiru S, Bante A, Molla A, et al. (2019) Neonatal sepsis and associated factors among newborns in hospitals of Wolaita Sodo Town, Southern Ethiopia. Research and Reports in Neonatology 9: 1-8.'

10. Giannoni E, Agyeman PKA, Stocker M, Posfay-Barbe KM, Heininger $U$, et al. (2018) Neonatal sepsis of early onset, and hospital-acquired and community-acquired late onset: 
A prospective population-based cohort study. J Pediatr 201: 106-114.e4.

11. Al-Matary A, Heena H, AISarheed AS, Ouda W, AIShahrani DA, et al. (2019) Characteristics of neonatal Sepsis at a tertiary care hospital in Saudi Arabia. J Infect Public Health 12: 666-672:

12. Jabiri A, Wella HL, Semiono A, Saria A, Protas J (2016) Prevalence and factors associated with neonatal sepsis among neonates in Temeke and Mwananyamala Hospitals in Dar es Salaam, Tanzania. Tanzania Journal of Health Research 18: 12-17.

13. Agnche Z, Yeshita HY, Gonete KA (2020) Neonatal sepsis and its associated factors among neonates admitted to neonatal intensive care units in primary hospitals in Central Gondar Zone, Northwest Ethiopia, 2019. Infect Drug Resist 13: 3957-3967.
14. Getabelew A, Aman M, Fantaye E, Yeheyis T (2018) Prevalence of neonatal sepsis and associated factors among neonates in neonatal intensive care unit at selected governmental hospitals in Shashemene Town, Oromia Regional State, Ethiopia, 2017. Int J Pediatr 2018: 7801272.

15. Siakwa M, Kpikpitse D, Mupepi SC, Semuatu M (2014) Neonatal sepsis in rural Ghana: A case control study of risk factors in a birth cohort.' International Journal of Research in Medical and Health Sciences 4: 72-83.

16. Ozkan H, Cetinkaya M, Koksal N, Celebi S, Hacımustafaoglu M (2014) Culture-proven neonatal sepsis in preterm infants in a neonatal intensive care unit over a 7 year period: Coagulase-negative Staphylococcus as the predominant pathogen. Pediatr Int 56: 60-66.

17. Dayoub A, Zaher A, Saleh L (2010) Meconium Amino fluid and its association to the early neonatal infection. Tishreen University Journal-Medical Sciences 32: 23-28. 